Western University

Scholarship@Western

Research Summaries

BrainsCAN

2018

\title{
The prefrontal cortex and obesity: a health neuroscience perspective
}

BrainsCAN, Western University

Cassandra J. Lowe

Western University

Amy C. Reichelt

Western University

Peter A. Hall

University of Waterloo

Follow this and additional works at: https://ir.lib.uwo.ca/brainscanresearchsummaries

\section{Recommended Citation}

BrainsCAN. "The prefrontal cortex and obesity: a health neuroscience perspective" (2018). Research Summaries. 1.

https://ir.lib.uwo.ca/brainscanresearchsummaries/1

This Book is brought to you for free and open access by the BrainsCAN at Scholarship@Western. It has been accepted for inclusion in Research

Summaries by an authorized administrator of Scholarship@Western. For more information, please contact tadam@uwo.ca, wlswadmin@uwo.ca. 


\section{The prefrontal cortex and obesity: a health neuroscience perspective}

\section{Background}

Humans have a strong and reliable preference for foods that are high in fat and sugar, which is thought to be an evolutionary outcome. As a species, we prefer to find and consume these kinds of foods.

The modern environment around us can be described as 'obesogenic' - it encourages unhealthy eating and poor lifestyle choices. Some modern foods are considered 'hyperpalatable' - so appealing that they seem to overpower our ability to control consumption. Trying to limit these calorie-rich food choices is partially dependent on the ability to override our internal desire, our gut reaction, to eat them.

\section{The Research}

The prefrontal cortex (PFC), the front region of the brain, is implicated in these kinds of internal desires, the intuitive or emotional choices we make. It is the area responsible for 'executive function' - the decision-making processes that choose between good and bad, conflicting thoughts, assessing future consequences of present actions, working towards goals, prediction and expectations.

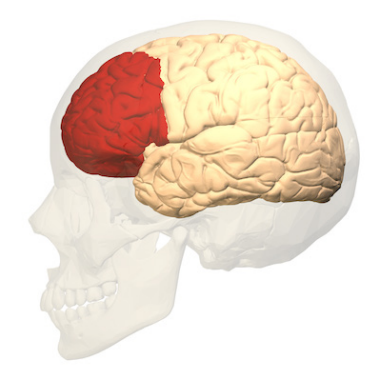

Figure 1 - prefrontal cortex

The ability to exert any control over these dietary desires for fatty and sugary food, what we call dietary self-regulation, varies among individuals.

Some current models of obesity have showed raised activity in certain reward centres of the brain in response to calorie-rich foods and that has lead to food-addiction models. Conversely, other models of obesity have come from reduced signaling in other reward regions in the brain, so that overeating is seen as an attempt to compensate for this reduced signaling.

Research has also shown that increased activity in a specific region of the PFC moderates food cravings and consumption of these hyperpalatable choices, in persons who would otherwise be susceptible to overconsumption. Reduced activity of the same region can increase the likelihood of overconsumption.
Key Points

The level of activity within an individual's prefrontal cortex seems to be critical to dietary self-control and the likelihood of overconsumption and obesity. Lower activity can make individuals more vulnerable to the appeal of calorie-rich foods. Sustained overconsumption and obesity can cause changes in the prefrontal cortex that further discourage dietary self-regulation, creating a reciprocal relationship that reinforces the poor dietary choices and encourages overconsumption.

\section{Publication}

Trends in Cognitive Sciences, February 2019 bit.ly/CALO0219-R

\section{BrainsCAN Support}

Postdoctoral Fellowship Program

Research Support

ARC

Western Faculty, Group or Institution

Brain and Mind Institute

\section{The Findings}

Our review used a health neuroscience framework to provide a comprehensive model centring on the PFC as a critical 
factor in someone's vulnerability to obesity. We explored how differences in the PFC structure and function are reflected in someone's dietary self-regulation abilities which can make obesity more likely, and how chronic consumption of calorie-dense food impacts reward sensitivity and functionality of the PFC that results in the deficits seen in persons with obesity.

Neuroimaging techniques, like fMRI, have helped link regional brain activity to an individual's behaviour and actions, including food choice-related self-control. Individual variations in the PFC structure and executive functioning can predispose an individual to overconsumption.

It has also been found that persons with obesity seem to be less able to recruit the areas of the PFC that modulate or downregulate food choices, compounding the challenge of dietary self-regulation. Studies have shown that across the lifespan, persons with obesity perform more poorly on various measures of cognition (such as memory and processing speed), suggesting that obesity itself results in certain cognitive impairments and suppressed executive functions. Reinforcing this finding, it has also been shown that behavioural and surgical weight-loss interventions can improve neurocognitive functioning and brain health.

We believe that the relationship between brain structure, function and obesity is most likely to be reciprocal in nature, rather than one leading to the other simply as cause-and-effect. Weaker PFC response to food cues may leave an individual more susceptible to the rewarding properties of calorie-rich foods. Reduced cognitive control over the reward regions of the brain reduces dietary self-regulation, leading to likely over-consumption in our modern obesogenic environment. Over time, repeated overconsumption further suppresses the PFC activity to food reward promoting further overconsumption, each reinforcing the other.

\title{
Next Steps
}

While the reciprocal relationship model we developed is grounded in theory, a long-term study is needed to successfully develop it further. How does this dietary self-regulation change over our lifespan? What lifestyle interventions (such as exercise) are most effective at optimising structure and function within the PFC and can these interventions encourage better dietary self-regulation?

\section{Western Researchers}

Cassandra J. Lowe

Amy C. Reichelt

\section{University of Waterloo Researcher}

\author{
Peter A. Hall
}

Figure credits

Figure 1 - Wikimedia Commons, CC BY-SA $2.1 \mathrm{jp}$ 